\title{
469 - A systematic review and meta-analysis of cognitive training in adults with Major Depressive Disorder
}

Claudia Woolf; Amit Lampit; Zeeshan Shahnawaz; Julieta Sabates; Louisa Norrie; David Burke; Sharon Naismith; Loren Mowszowski

Major depressive disorders (MDD) are common and disabling, and are linked to functional impairment and increased mortality. While current treatments for MDD are moderately effective, ultimately, up to one third of patients do not achieve full remission. Interestingly, while affective symptoms of depression resolve with the resolution of a depressive episode, cognitive impairment frequently persists, and has been identified as one of the most prominent predictors of illness recurrence. Additionally, MDD is wellrecognised as a key risk factor for further cognitive decline and dementia. Yet, available treatments in MDD do not typically address cognitive impairment. Cognitive training (CT), represents a promising and novel therapeutic intervention in this regard. Our review systematically identified and quantitatively evaluated the evidence for CT in adults with MDD. Following PRISMA guidelines, eligible studies were selected according to pre-defined criteria delineating our target population (adults with clinically defined MDD), parameters for CT interventions (computer-or strategy-based, clinician-facilitated), and study design (controlled trials including pre/post cognitive and psychological or functional outcome data). Of 111 identified, nine studies met inclusion criteria. These studies were evaluated for methodological quality and risk of bias. Despite heterogeneity, qualitative and meta-analytic synthesis of study findings revealed significant improvements in cognitive and affective outcomes following $\mathrm{CT}$, with pooled effect sizes ranging from moderate to large. Unfortunately, very few studies investigated 'far transfer' to broader domains of everyday functioning. Overall, given the strong evidence supporting the efficacy and value of CT in this context, CT should be considered as a primary therapeutic intervention in the holistic treatment of MDD. 